\section{Routine Replacement versus Clinical Monitoring of Peripheral Intravenous Catheters in a Regional Hospital in the Home Program: A Randomized Controlled Trial}

\author{
Patricia Van Donk, NP, GradDip(AdvClinPrac), MN; \\ Claire M. Rickard, BN, GradDip(CritCare), PhD; \\ Matthew R. McGrail, BSc, GradDip(IT), PhD; \\ Glenn Doolan, BAppSc(AdvNurs), MNA
}

This randomized, controlled trial involving 316 patients in the home setting found no difference in the rate of phlebitis and/or occlusion among patients for whom a peripheral intravenous catheter was routinely resited at 72-96 hours and those for whom it was replaced only on clinical indication (76.8 events per 1,000 device-days vs 87.3 events per 1,000 device-days; $P=.71$ ). There were no bloodstream infections.

Infect Control Hosp Epidemiol 2009; 30:915-917

Routine replacement of peripheral intravenous devices (IVs) every 72-96 hours was recommended by the Centers for Disease Control (CDC) in 2002 to avoid phlebitis (not infection) in adults but not recommended in pediatric patients or adults with poor veins. ${ }^{1}$ However, routine replacement is often ignored if patients are to be discharged from the hospital soon or if resite is thought to be difficult. ${ }^{2}$ The CDC cites one nonrandomized study in which IVs were associated with equivalent rates of phlebitis when left in situ for either 72 or 96 hours. ${ }^{3}$ Two recent randomized, controlled trials involving hospital inpatients found no difference in rates of phlebitis when IVs were resited every third day or only on clinical indication. ${ }^{4,5}$ Maki $^{6}$ questioned the generalizability of results to hospitals without specialized IV teams, although $26 \%$ of IVs in the larger study were inserted by staff nurses and doctors, not the IV service. ${ }^{4}$ Our aim was to test routine replacement in community-based Hospital in the Home patients, with IVs inserted by staff doctors and nurses, not an IV team.

\section{METHODS}

After approval by an ethics committee, this randomized controlled trial was conducted during 18 months in the Hospital in the Home service of a regional hospital. Adult patients who were referred by their family doctor or the emergency department and who were prescribed IV antibiotics were approached for informed consent. Figure 1 details patient flow. Randomization allocation was concealed until treatment via sealed opaque envelopes. IVs were routinely resited at 72-96 hours (for control subjects) or only on clinical indication (for case patients). All IVs were inspected daily and removed for phlebitis or occlusion or if no longer required. Blood cultures were performed if patients showed signs of bloodstream infection. IVs were inserted by emergency department or family doctors and emergency department and/or Hospital in the Home nurses. Insertion and care techniques were consistent with CDC recommendations. ${ }^{1}$

Phlebitis and occlusion were assessed daily by 6 Hospital in the Home registered nurses, overseen by the principal investigator (P.V.D.). Phlebitis was defined as a total score of 2 or more points from the following factors: pain (on a 10point scale, $1=1$ point, and 2 or more $=2$ points); redness (less than $1 \mathrm{~cm}=1$ point, and 1 or more $\mathrm{cm}=2$ points); swelling (as for redness); and discharge (hemoserous ooze under dressing $=1$ point, and hemoserous ooze requiring dressing change or purulence $=2$ points). Occlusion was defined as the inability to inject or aspirate. Compared with other definitions that usually require at least 2 symptoms (eg, pain and redness), ${ }^{7}$ our definition was similar but more conservative. In clinical practice, IVs are removed if one symptom alone is severe enough, and we were mindful that registered nurses were only intermittently in the home.

Power calculations indicated that an enrollment of $96 \mathrm{pa}-$ tients per group would give the study an $80 \%$ power to detect a risk ratio of $1.5(\alpha=.05)$. The primary outcome was the combined rate of phlebitis and occlusion per 1,000 devicedays (with a 95\% confidence interval [CI]). Incidence between groups was compared using a Kaplan-Meier survival curve (with log rank Mantel-Cox test). Multivariate (Cox regression) modelling assessed possible associations with the phlebitis and/or occlusion. We used an intention-to-treat analysis, and $P<.05$ was considered to be significant.

\section{RES ULTS}

One hundred five patients (representing 155 IVs and 16,765 IV-hours) underwent resite of IVs only on clinical indication, and 95 patients (representing 161 IVs and 12,192 IV-hours) underwent resite of IVs routinely. As expected, the case patient group had a longer IV dwell time (mean, 108.2 hours vs 75.7 hours) and fewer IVs per person (mean, 1.5 vs 1.7 ). The longest IV dwell time for a case patient was 19 days, but $52 \%$ of the IVs were removed by 96 hours and $85 \%$ by 7 days. No physical deterioration in IVs over time was observed. Compliance with routine resite in control subjects was $77 \%$. Across both groups, $21 \%$ of patients did not complete the study protocol for all IVs, usually when patients opted for single-use "butterfly" needles rather than resite.

Diagnoses were commonly cellulitis or pneumonia, followed by wound, bursitis, sinus, urinary and/or kidney in- 


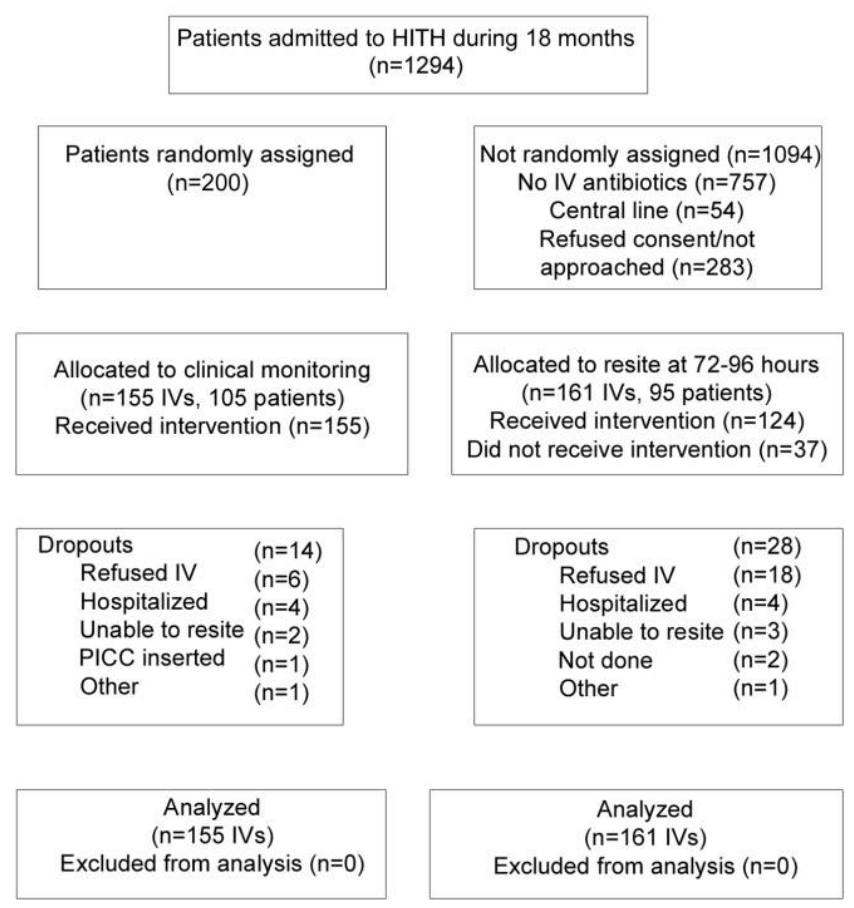

FIGURE 1. Flow chart of participants through a randomized, controlled trial in the home setting designed to compare rates of phlebitis and/or occlusion in patients for whom a peripheral intravenous catheter was routinely resited at 72-96 hours and those for whom it was replaced only on clinical indication. HITH, Hospital in the Home.

fections, and other infections. Treatment, in order of descending frequency, was once-daily cephazolin and probenecid, daily ceftriaxone, twice-daily cephazolin, flucloxacillin, vancomycin, gentamicin, penicillin, or other antibiotics. Fifty-eight percent of the patients were male, and the mean age was 59 years. The mean gauge for the IV was 20-22 g. IVs were inserted in the forearm (52\%), hand (19\%), cephalic fossa and/or wrist (15\%), antecubital fossa (11\%), or unknown (3\%). Most IVs (79\%) were inserted by nurses, $11 \%$ by doctors, and $10 \%$ by unknown. Skin preparation was by $70 \%$ alcohol swab (50\%), $70 \%$ alcoholic chlorhexidine swab (35\%), or unknown (15\%). Inserters used soap and water handwash (44\%), alcohol-based handrub (35\%), or unknown (21\%). The only significant baseline difference was that case patients were a mean of 8 years older $(P=.002)$ and more likely to have inserters use soap and water handwash than alcohol-based handrub $(P<.03)$.

Phlebitis was associated with 79 IVs and occlusion with 21 IVs. There were 61 phlebitis and/or occlusion events among case patients and 39 events among control subjects. This equated to respective rates of 87.3 events per 1,000 catheterdays ( $95 \%$ CI, 65.4-109.2 events per 1,000 catheter-days) and 76.8 events per 1,000 catheter-days (95\% CI, 52.7-100.9 per $1,000$ catheter-days $)(P=.71$; Kaplan-Meier with log-rank $)$ (Figure 2). Most phlebitis was associated with pain, redness, or swelling. Only $11 \%$ of the cases of phlebitis were associated with discharge, and this was always hemoserous. If a "2symptom, any severity" criteria had been used for phlebitis, the rate would have been reduced by one-third. No purulence or bacteremia occurred. Four patients per group required hospitalization, which was unrelated to their IVs.

Independent of study group, the rate of phlebitis and/or occlusion at 96 hours was $23.4 \%$ ( 74 of 316 IVs), which was identical to the rate of phlebitis and/or occlusion associated with the 111 IVs used beyond 96 hours $(P=.99)$. The incidence of phlebitis and/or occlusion differed significantly across days 1 to $7(P=.02)$, but there was no temporal pattern of increasing or decreasing incidence with time. The final multivariate model found only 2 significant variables associated with phlebitis and/or occlusion: female sex $(P=$ $.003)$ and insertion by emergency department doctors $(P=$ $.024)$. Study group was not significant on any analysis.

\section{I S C US S I O N}

We found no difference in the rate of complications when IVs were resited every 72-96 hours or only on clinical indication. The results are consistent with recent hospital randomized, controlled trials and provide further evidence against routine IV replacement, in this case for community patients for whom IVs were not inserted by a specialized IV team but by community and emergency department staff.,

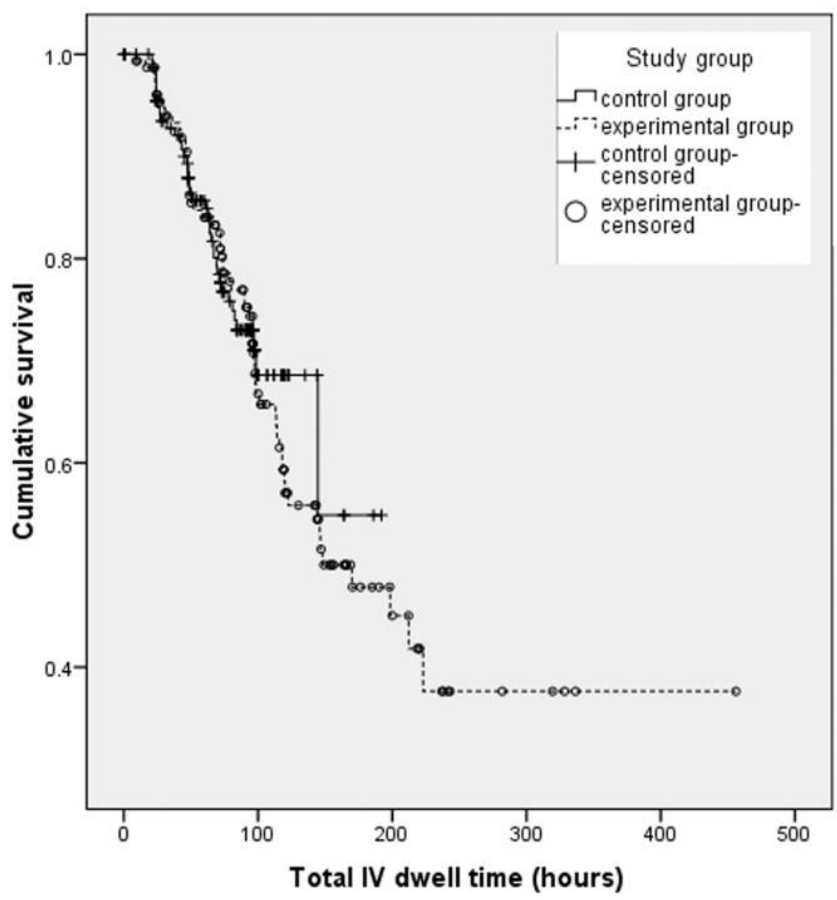

FIGURE 2. Graph comparing intravenous catheter (IV) survival from phlebitis and/or occlusion for control subjects with that of case patients (experimental group). There was no significant difference $(P=.71 ; \log$ rank Mantel-Cox test). 
Case patients were statistically 8 years older than control subjects and more likely to have inserters use soap and water handwash than alcohol-based handrub. We doubt this affected risk between groups. ${ }^{8}$ We saw higher rates of phlebitis in females, as have other studies; this may be linked to biological or behavioral factors. ${ }^{7,9,10}$ Emergency department doctors had significantly higher complication rates than other inserters, but absolute numbers from this group were small $(n=11)$.

The lowest rate of phlebitis occurred on day 1 ; this fact suggests that the body takes this long to mount a detectable response. We modeled a range of scenarios (data not shown) from the observed phlebitis incidence per day, with theoretical routine resite at $24,48,72$, and 96 hours over a week's treatment. Only routine resite every 24 hours would have reduced complications; this frequency would be unfeasible in terms of staff time, patient discomfort, and IV access sites. Maki and Ringer ${ }^{7}$ also noted that their observational data supported daily resite but did not recommend this as a routine.

Ideally, we would have had a blinded phlebitis assessor and routine blood and device cultures, but this was not possible with our resources. We believe that data collectors were consistent because they uniformly asked patients about pain on a 10-point scale and measured redness and swelling with disposable tape measures and because discharge and occlusion are obvious clinical signs. All patients received IV antibiotics that may have protected against infection but conversely have increased the risk of vein irritation. We used the patient, not the IV, as the unit of measurement, and we considered the vital factor of time. Crude comparisons of the percentage of IVs associated with phlebitis unfairly give an advantage to the routine replacement group because exposure to risk is reduced. Per patient comparisons are also flawed unless time is considered. Survival analysis of rates per 1,000 device-days allows a true comparison between different care regimes. A larger study would be needed to confirm our observed incidence rates and rule out significant differences at these levels.

IV insertion is unpleasant and expensive. Resite only on clinical indication spares patients from repeated insertions and allows redirection of scarce health funding and staff. In our department, resite on clinical indication would save A $\$ 20,000$ (US\$15,800) annually (IV and insertion kit at A \$6 [US\$4.74] each plus registered nurse insertion time of 15 minutes). The trial supports indefinite IV dwell time in the home setting as long as IVs are monitored daily and removed promptly if complications occur.

\section{ACKNOWLEDGMENTS}

We are grateful to the management, staff, and patients of the Hospital in the Home program and Emergency Department of the Latrobe Regional Hospital, who supported the study, and Gillian Ray-Barruel, who provided assistance in editing the manuscript.

Potential conflicts of interest. All authors report no conflicts of interest relevant to this article.

From Latrobe Regional Hospital, Traralgon (P.V.D.), and the Department of Medicine, Nursing, and Health Sciences (G.D.) and the School of Rural Health (M.R.M.), Monash University, Gippsland Campus, Victoria; and the Research Centre for Clinical and Community Practice Innovation (C.M.R.) and the School of Nursing and Midwifery (C.M.R.), Griffith University, Nathan, Queensland, Australia.

Address reprint requests to Patricia Van Donk, RN, Latrobe Regional Hospital, Princes Hwy, Traralgon, Victoria 3844, Australia (TVanDonk@lrh .com.au).

Received December 15, 2008; accepted April 2, 2009; electronically published July 28, 2009.

(C) 2009 by The Society for Healthcare Epidemiology of America. All rights reserved. 0899-823X/2009/3009-0018\$15.00. DOI: 10.1086/599776

\section{REFERENCES}

1. O'Grady NP, Alexander M, Dellinger EP, et al. Guidelines for the prevention of intravascular catheter-related infections. MMWR Recomm Rep 2002; 51(RR-10):1-29.

2. Schultz AA, Gallant P. Evidence-based quality improvement project for determining appropriate discontinuation of peripheral IV cannulas. Evid Based Nurs 2005; 8:8.

3. Lai KK. Safety of prolonging peripheral cannula and IV tubing use from 72 to 96 hours. Am J Infect Control 1998; 26:66-70.

4. Webster J, Clarke S, Paterson D, et al. Routine care of peripheral intravenous catheters versus clinically indicated replacement: randomised controlled trial. BMJ 2008; 337:a339.

5. Webster J, Lloyd S, Hopkins T, Osborne S, Yaxley M. Developing a research base for intravenous peripheral cannula resites (DRIP trial): a randomised controlled trial of hospital inpatients. Int J Nurs Stud 2007; 44: 664-671.

6. Maki DG. Improving the safety of peripheral intravenous catheters: specialized teams could bring greater benefit than routine replacement. $B M J$ 2008; 337:a630.

7. Maki DG, Ringer M. Risk factors for infusion-related phlebitis with small peripheral venous catheters. Ann Intern Med 1991; 114:845-854.

8. Hirschmann H, Fux L, Podusel J, et al; EURIDIKI (European Interdisciplinary Committee for Infection Prophylaxis). The influence of hand hygiene prior to insertion of peripheral venous catheters on the frequency of complications. J Hosp Infect 2001; 49:199-203.

9. Martinez JA, Fernández P, Rodríguez E, et al. Intravenous cannulae: complications arising from their use and analysis of predisposing factors. Med Clin (Barc) 1994; 103:89-93.

10. Kagel EM, Rayan GM. Intravenous catheter complications in the hand and forearm. J Trauma 2004; 56:123-127. 